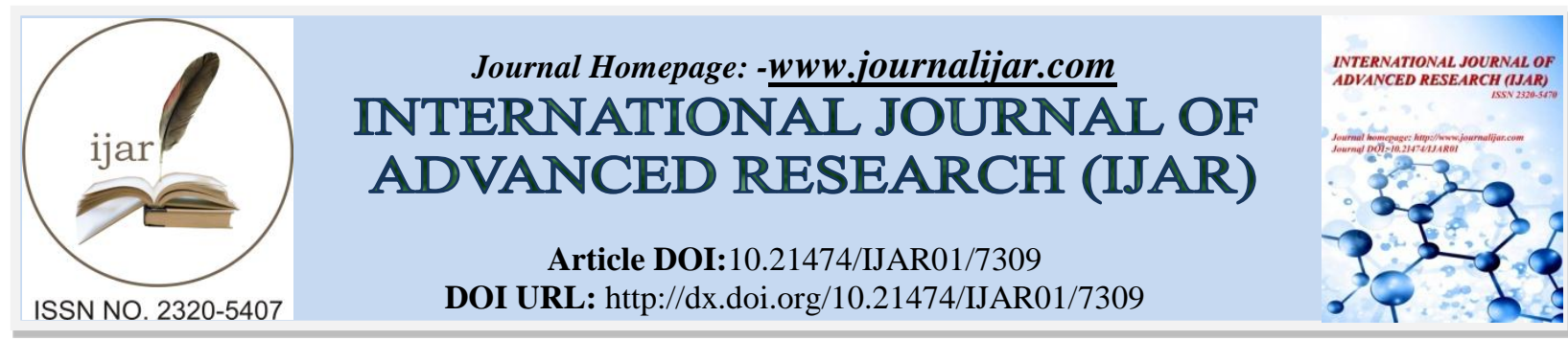

RESEARCH ARTICLE

\title{
PREVALENCE OF OBSTRUCTIVE SLEEP APNEA (OSA) RISK AND SLEEP QUALITY OF LIFE (SQOL) AMONG COPD PATIENTS OF A TERTIARY CARE HOSPITAL, LUDHIANA, PUNJAB.
}

\author{
Gaganpreet Kaur ${ }^{1}$, Bindu K ${ }^{2}$, Prabhjot Saini, Anil Kumar Kashyap. \\ 1. College of Nursing, Dayanand Medical College \& Hospital, Ludhiana. \\ 2. Associate Professor, Dept. of Medical Surgical Nursing, College of Nursing, Dayanand Medical College \& \\ Hospital, Ludhiana. \\ 3. Professor, Dept. of Medical Surgical Nursing, College of Nursing, Dayanand Medical College \& Hospital, \\ Ludhiana. \\ 4. Associate Professor, Dept. of Pulmonary Medicine, Dayanand Medical College \& Hospital, Ludhiana.
}

\section{Manuscript Info}

(.........................

Manuscript History

Received: 17 April 2018

Final Accepted: 19 May 2018

Published: June 2018

Keywords:-

Obstructive sleep apnea risk, sleep quality of life, COPD patients.

\section{Abstract}

Background: Chronic Obstructive Pulmonary Disease (COPD) is one of the most common and serious disorder which not only affect the individual physically but also the quality of life of the person to a higher extent. Obstructive sleep apnea (OSA) syndrome and (COPD) are two diseases that often coexist within an individual. Sleep-related disturbances and insomnia have been shown to be higher in COPD patients. Objective: The present study was conducted with an objective to assess the prevalence of obstructive sleep apnea risk and sleep quality of life among COPD patients.Methodology: A descriptive study was conducted on 100 stable COPD patients at medical OPD of DMC \& Hospital, Ludhiana. The subjects were selected by convenience sampling technique. Modified Berlin questionnaire was used to check OSA risk and Quebec sleep questionnaire was used for assessing SQOL among the subjects. Data was collected by self-report method.Results: The findings revealed that $72 \%$ of COPD patients (Mean age $57.1 \pm 1.70$ ) were at high risk of OSA while $68 \%$ showed average sleep quality of life. There was a significant association between OSA risk and SQOL among COPD patients $(\mathrm{p}=0.00)$. OSA risk was also associated with married $(\mathrm{p}=0.03)$, non working $(0.01)$, presence of chronic illness $(\mathrm{p}=0.00)$, obese $(\mathrm{p}=0.00)$ and bigger neck circumference $(\mathrm{p}=0.00)$.Conclusion: It was concluded that there was high risk of obstructive sleep apnea in COPD patients and average sleep quality of life. There is a significant association of OSA risk and sleep quality of life among COPD patients. Therefore a protocol should be made to screen the COPD patients for OSA risk.

Copy Right, IJAR, 2018,. All rights reserved.

\section{Introduction:-}

Respiratory conditions affect the airways, including the lungs as well as the passages that transfer air from the mouth and nose into the lungs. They can be long lasting (chronic) or short term (acute) and can cause ill health, disability 
and death. Respiratory conditions are believed to be the most commonly managed problems in general practice. Data from the Bettering the Evaluation and Care of Health survey of general practitioners suggest that they were managed at a rate of approximately 20 times per 100 encounters from 2003-04 to 2012-13 (Britt 2013). ${ }^{1}$

Chronic Obstructive Pulmonary Disease (COPD) is a progressive inflammatory disease characterized by chronic obstruction in the peripheral bronchus and pulmonary emphysema. These diseases are disabling with symptoms such as chronic cough, phlegm, wheezing, shortness of breath and increased infections of the respiratory passage. These conditions affect the quality of life of the individual to the great extent, in which sleep disturbance is the important manifestation which need to be controlled or treated. ${ }^{2}$

Obstructive sleep apnoea (OSA) syndrome is a common and often life-altering sleep-related breathing disorder. It not only adversely affects physical health, but the quality of life of these patients is also often significantly compromised. They experience excessive daytime sleepiness and poor cognitive, social and exercise performance. ${ }^{3}$ It has been noted that sleep disturbances affect the life of the individual in respiratory conditions mainly COPD due to OSA. In view of this, a descriptive study is planned to assess the prevalence of obstructive sleep apnoea risk and sleep quality of life among COPD patients.

Moreover, In Indian scenario there are very few studies conducted on the prevalence of obstructed sleep apnea and associated sleep quality of life among COPD patients. Therefore, a descriptive study was planned to assess the prevalence of obstructive sleep apnoea risk and sleep quality of life among COPD patients.

\section{Material and Methods:-}

This was a descriptive study carried out at medical OPD of DMC \& Hospital, Ludhiana. Stable COPD patients coming to medical OPD of DMCH who were diagnosed with COPD for more than 1 year were selected as the study sample. Convenience sampling technique was used and sample size was 100. Data was collected by self report method of data collection. Tool used for data collection included socio demographic profile, (age, gender, marital status, religion, working status, habitat, family income and socio-economic status, smoking and alcohol habits) clinical profile sheet, (Diagnosis of patient, Stages of COPD, Duration of illness, presence of any other chronic illness and vital signs of the patient, anthropometric measurements of the patients which includes BMI, waist hip ratio, neck length and neck circumference) Modified Berlin questionnaire, Quebec sleep questionnaire.

All data was entered in Microsoft excel and descriptive and inferential statistics was used for analysis of the data.

\section{Analytical Approach:-}

Statistical analysis was done using the Statistical Package for Social Sciences (SPSS) 17.0. Numerical data were summarized using means and standard deviation, while categorical data were summarized using frequencies and percentages. Comparison between groups was done usingChi-square test.

\section{Results:-}

The mean age of COPD patients was57.1 \pm 1.70. Out of 100 COPD patients maximum number of patients were in age group of 51-70 yrs, males, married, belong to hindu religion, obtained elementary education, working, residing in the urban area and there was no remarkable difference between the dietary pattern of the patients. Near about half of patients were from middle/lower middle class III according to Kuppuswamy's SES. Percentage distribution of COPD patients as per smoking habits and $48 \%$ were non-smokers while $52 \%$ were smokers. Percentage distribution of COPD patients as per alcohol consumption and 55\% were alcoholic, while $45 \%$ were non alcoholic. the percentage distribution of COPD patients as per clinical profile. Out of 100 subjects $2 / 3^{\text {rd }}(67 \%)$ were suffering from COPD $\leq 15$ yrs and $1 / 3^{\text {rd }}(33 \%)$ of the patients were suffering from COPD $>15 \mathrm{yrs} .2 \%$ of the patients was found in mild stage of COPD, $76 \%$ of the patient were found to be in moderate and $22 \%$ of the patients were in severe stage of COPD. Out of 100 COPD patients $60 \%$ of the patients were under treatment from $\leq 10$ years, $40 \%$ of the patients were under treatment from $>10$ years. Among the subjects $63 \%$ of the patients were also suffering from other chronic illness along with COPD (diabetes mellitus 63.5\%, hypertension 63.5\%, coronary artery disease $23.8 \%$, viral hepatitis $7.9 \%$ ) and $37 \%$ of the patients were without any chronic illness. 
Table 1:-Sociodemographic variables of COPD patients $\mathrm{N}=100$

\begin{tabular}{|c|c|}
\hline \multirow{3}{*}{$\begin{array}{l}\text { Sociodemographic Characteristics } \\
\text { Age (in yrs)* } \\
\quad 31-50\end{array}$} & \multirow[t]{2}{*}{$\mathbf{f}(\%)$} \\
\hline & \\
\hline & 17 \\
\hline $51-70$ & 83 \\
\hline \multicolumn{2}{|l|}{ Gender } \\
\hline Male & 75 \\
\hline Female & 25 \\
\hline \multicolumn{2}{|l|}{ Marital status } \\
\hline Married & 83 \\
\hline Divorced/ Widow/ Widower & 17 \\
\hline \multicolumn{2}{|l|}{ Religion } \\
\hline Sikh & 28 \\
\hline Hindu & 72 \\
\hline \multicolumn{2}{|l|}{ Educational status } \\
\hline Illiterate & 08 \\
\hline Elementary & 57 \\
\hline Senior secondary & 16 \\
\hline Graduate \& above & 19 \\
\hline \multicolumn{2}{|l|}{ Working status } \\
\hline Working & 54 \\
\hline Non working & 46 \\
\hline \multicolumn{2}{|l|}{ Occupation $(n=54)$} \\
\hline Industrial worker & $02(03.7)$ \\
\hline Businessman & $21(38.9)$ \\
\hline Labourer & $08(14.8)$ \\
\hline Govt employee & $10(18.5)$ \\
\hline Farmer & $11(20.4)$ \\
\hline Doctor & $02(03.7)$ \\
\hline \multicolumn{2}{|l|}{ Habitat } \\
\hline Rural & 34 \\
\hline Urban & 66 \\
\hline \multicolumn{2}{|l|}{ Dietary pattern } \\
\hline Vegetarian & 43 \\
\hline Non-vegetarian & 42 \\
\hline Lacto ovo vegetarian & 15 \\
\hline \multicolumn{2}{|c|}{ Socio-economic status (as per kuppuswamy's SES) } \\
\hline Upper class I & 06 \\
\hline Upper middle class II & 39 \\
\hline Middle/lower middle class III & 48 \\
\hline Lower/upper lower class IV & 07 \\
\hline
\end{tabular}




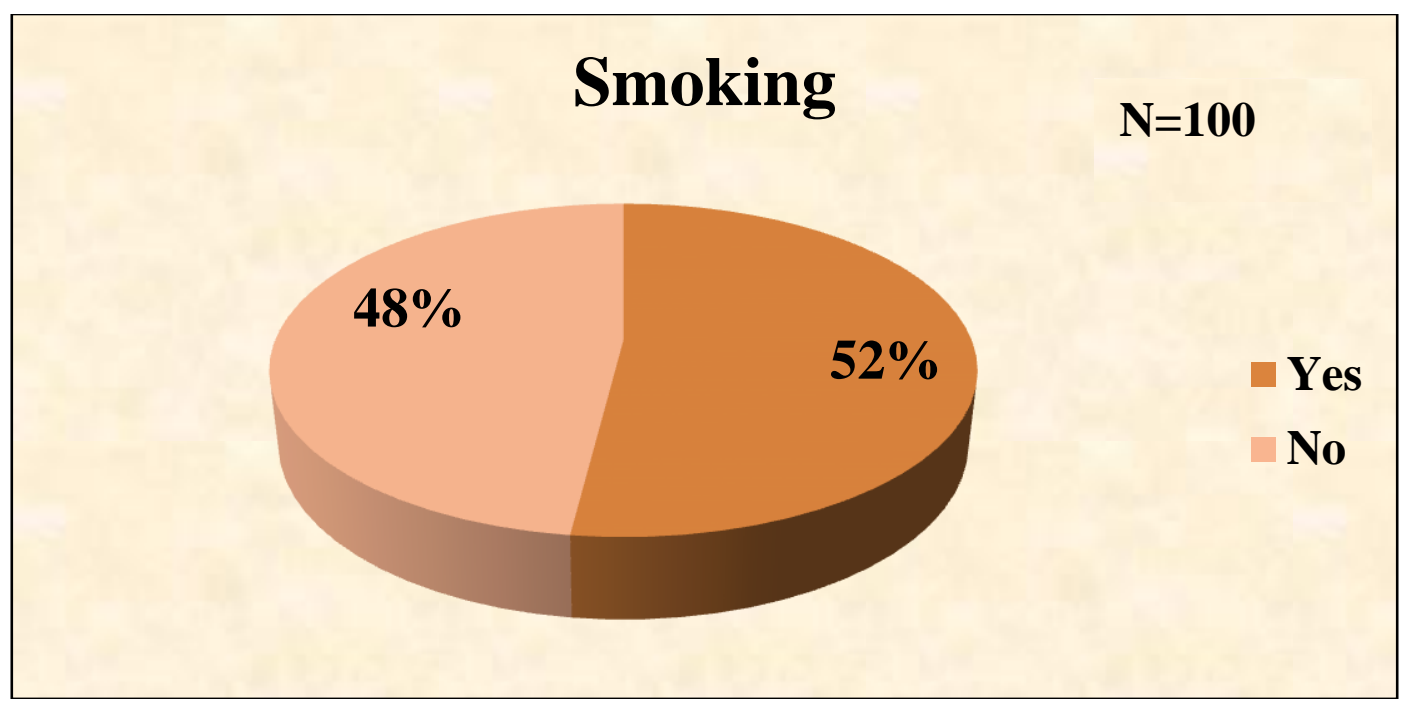

Figure 1:-Smoking habits among COPD patients

Figure 1depicts the percentage distribution of COPD patients as per smoking habits and $48 \%$ were non-smokers while $52 \%$ were smokers.

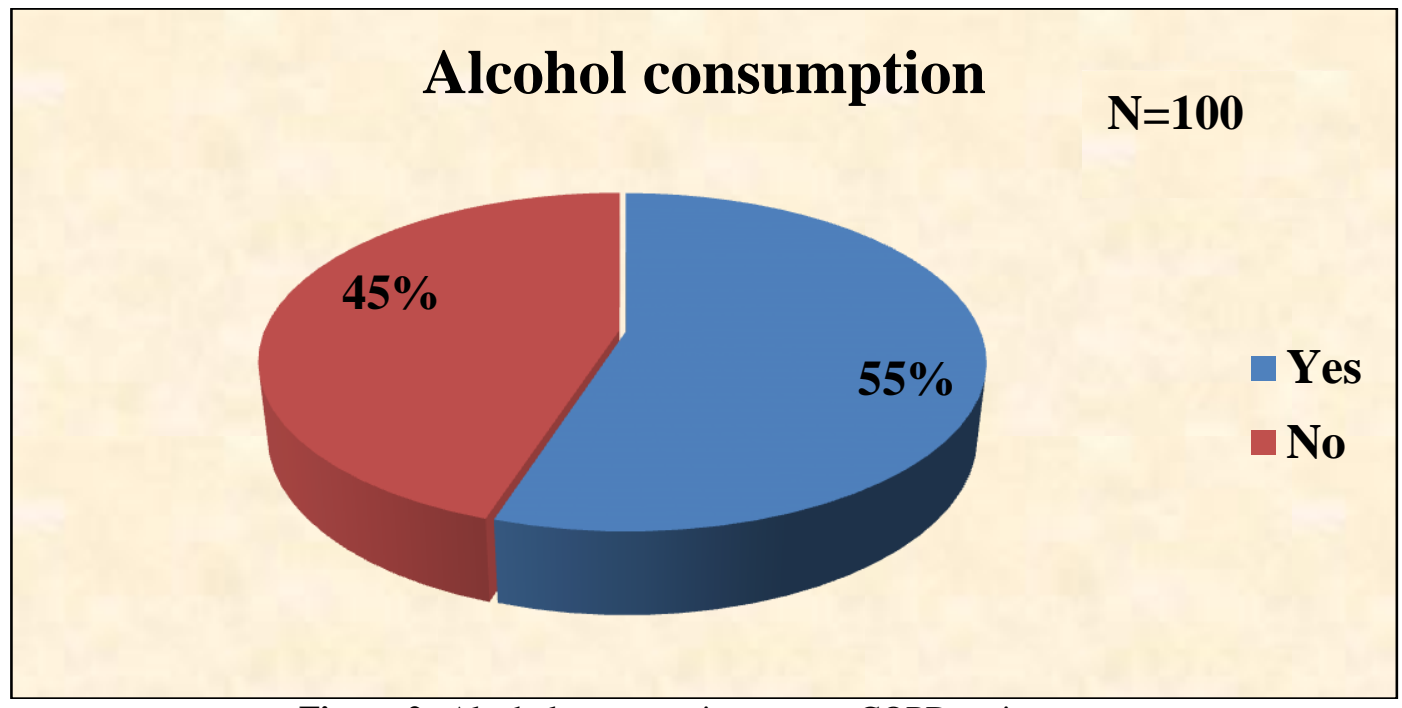

Figure 2:-Alcohol consumption among COPD patients

Figure 2 depicts the percentage distribution of COPD patients as per alcohol consumption and 55\% were alcoholic, while $45 \%$ were non alcoholic.

The percentage distributions of the COPD patients as per anthropometric measurements. Among 100 COPD patients $42 \%$ were obese, $26 \%$ were overweighted, $25 \%$ were normal and only $7 \%$ of the patient were underweighted. Waist hip ratio shows that $39 \%$ were at moderate risk, $33 \%$ were at high risk and $28 \%$ were at low risk of developing diseases. It also shows that $72 \%$ of the patients were having neck length between 5-8 cms and $28 \%$ with neck length between 1-4 cms. Among the subjects $62 \%$ of the patients were having neck circumference between $41-45 \mathrm{cms}$, $27 \%$ were having between $36-40 \mathrm{cms}$ and only $11 \%$ were with $31-35 \mathrm{cms}$. the percentage distributions of the COPD patients as per anthropometric measurements. Among 100 COPD patients $42 \%$ were obese, $26 \%$ were overweighted, $25 \%$ were normal and only $7 \%$ of the patient were underweighted. Waist hip ratio shows that $39 \%$ were at moderate risk, $33 \%$ were at high risk and $28 \%$ were at low risk of developing diseases. It also shows that $72 \%$ of the patients were having neck length between $5-8 \mathrm{cms}$ and $28 \%$ with neck length between 1-4 cms. Among 
the subjects $62 \%$ of the patients were having neck circumference between $41-45 \mathrm{cms}, 27 \%$ were having between $36-$ $40 \mathrm{cms}$ and only $11 \%$ were with $31-35 \mathrm{cms}$.

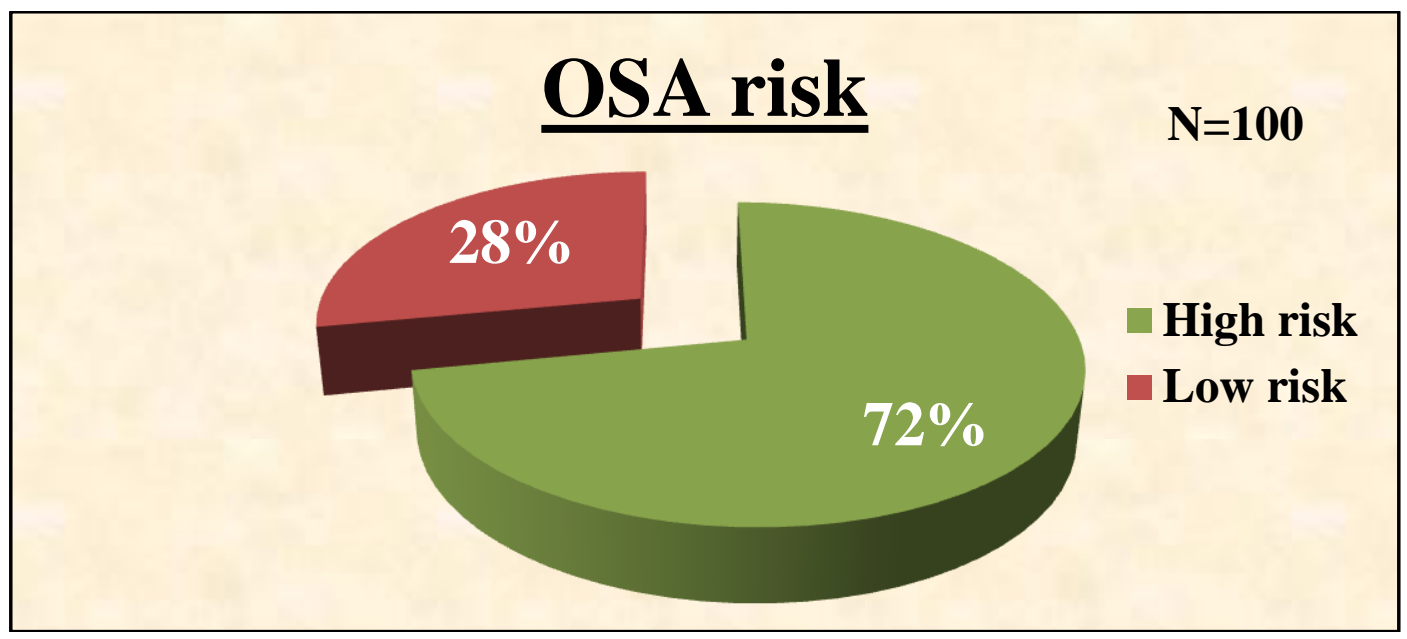

Figure 3:-Prevalence of OSA risk among COPD patients

Figure 3 reveals the prevalence of obstructive sleep apnea risk among COPD patients. This study showed that $72 \%$ of COPD patients were at high risk for developing obstructive sleep apnea while only $28 \%$ of COPD patients were at low risk of OSA.

Hence it was concluded that COPD patients were significantly at high risk of developing OSA.

The sleep quality of life (SQOL) among COPD patients. It revealed that $68 \%$ of COPD patients had average SQOL with mean score $126.63 \pm 12.30$ while $32 \%$ of COPD patientshad good SQOL with mean score $173.65 \pm 14.84$. Therefore, it was concluded that SQOL was altered in COPD patients.

Table 2:-Association of obstructive sleep apnea risk with sleep quality of life among COPD patients.

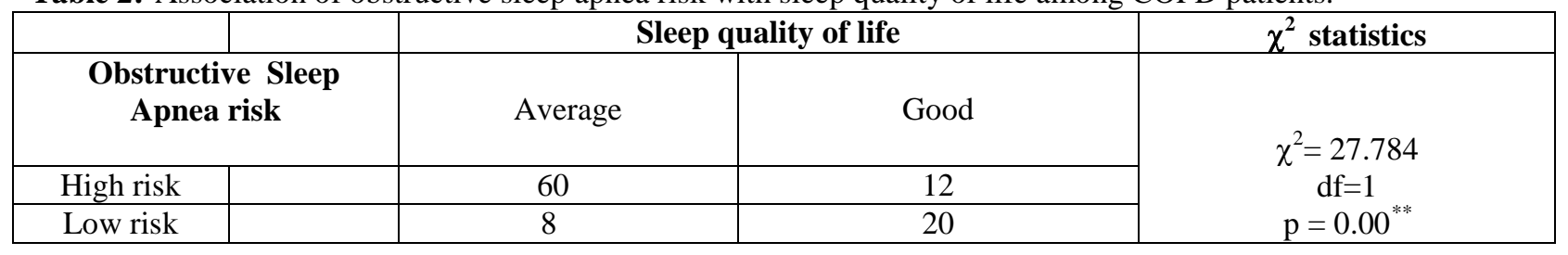

This table shows the association of obstructive sleep apnea risk with sleep quality of life among COPD patients. Findings showed that out of 100 stable COPD patients, $60 \%$ patients of high risk obstructive sleep apnea had average sleep quality of life while only $12 \%$ showed good sleep quality of life as compared to $8 \%$ low risk group had average sleep quality of life while $20 \%$ had good sleep quality of life. Therefore, it is concluded that obstructive sleep apnea risk is significantly associated with sleep quality of life. $(\mathrm{P}=0.00)$. There was a significant association of gender $(\mathrm{p}=0.01)$, marital status $(\mathrm{p}=0.03)$, working status $(\mathrm{p}=0.01)$, smoking $(\mathrm{p}=0.02)$ and alcohol consumption $(\mathrm{p}=0.01)$ with obstructive sleep apnea risk, while no statistical significance found with age and socio economic status ( $\mathrm{p}>0.05$ ). factors like duration of treatment and presence of other chronic illness were found out be to be statistically associated with obstructed sleep apnea risk among COPD patients. ( $\mathrm{p}<0.05$ ). factors like BMI and neck circumference were found to be statistically associated with obstructive sleep apnea risk of COPD patients $(\mathrm{p}<0.05)$

\section{Discussion:-}

Obstructive sleep apnea (OSA) is a common sleep disorder characterized by intermittent partial or complete upper airway obstruction during sleep, leading to intermittent hypoxemia, recurrent arousals, sleep fragmentation and poor sleep quality. The prevalence of OSA with accompanying daytime sleepiness is approximately 3 to $7 \%$ for adult men and 2 to $5 \%$ for adult women in the general population. 
Patients with OSA may present several typical symptoms including habitual snoring, feeling of unrefreshed awaking, excessive daytime sleepiness (EDS), fatigue, lack of concentration, memory impairment, and at times psychological disturbances.

Chronic Obstructive Pulmonary Disease (COPD) is very common disease leading to frequent physician visits and hospitalizations and become the $3^{\text {rd }}$ leading cause of death. Sleep related disturbances like OSA is very common among COPD patients. OSA occurs in about 10 to $15 \%$ of patients with COPD, a condition referred to as the "overlap syndrome". Although the prevalence of OSA is similar in patients with COPD as in the general population, individuals with both conditions without Continuous Positive Airway Pressure (CPAP) treatment have an increased risk of death and more hospitalizations from acute exacerbations.

As OSA in COPD can cause various serious complications, it also affects the quality of life of the individual. So researcher has planned to conduct a descriptive study to assess the prevalence of obstructive sleep apnea risk and sleep quality of life among COPD patients of a tertiary care hospital, Ludhiana, Punjab.

The analysis of socio demographic profile of COPD patients revealed that out of 100 subjects, $83 \%$ of COPD patients were in age group 51-75 years, 75 were males, $83 \%$ were married, $57 \%$ were educated up to elementary level, $54 \%$ were working, $72 \%$ were Hindu, $66 \%$ were residing in urban area, and $48 \%$ were in middle/lower middle class.

According to their personal habits related to smoking and alcohol consumption 52\% of COPD patients were smokers and 55\% were alcoholic. As per their clinical profile $67 \%$ were having COPD $\leq 15$ years, $78 \%$ of the patients were in moderate stage of illness, $60 \%$ of the patients were undergoing treatment from $\leq 10$ years and $63 \%$ of the patients were suffering from another chronic illness along with COPD.

According to the vital signs of the patients, $58 \%$ were having normal heart rate, $85 \%$ were having abnormal respiratory rate, $79 \%$ of the patients were having normal body temperature and $62 \%$ of the patients were having normal blood pressure.

Atlast according to the anthropometric measurements, $42 \%$ of the COPD patients were obese, $39 \%$ of the patients were having moderate risk according to the waist hip ratio, $72 \%$ of the patients were having neck length of 5-8 $\mathrm{cms}$ and $62 \%$ of them were neck circumference of $41-45 \mathrm{cms}$.

The present study revealed that maximum subjects (72\%) had high risk of developing obstructive sleep apnea whereas only $28 \%$ of subjects had low risk of developing obstructive sleep apnea.

This finding was supported by Feinsilver Steven et.al (2010) who conducted a prospective observational study in USA to determine whether reduced pulmonary function in obstructive airway disease (OAD) is an independent risk factor for obstructive sleep apnea. Berlin Questionnaire (BQ) was used. The prevalence of patients with a high risk of OSA was $55.2 \%$ in the OAD group, which was higher than in family members without history of $\mathrm{OAD}(7.5 \%, \mathrm{p}<$ $0.0001)^{4}$

It was also supported by a study conducted by Golshan Mohammad et. al (2010)on epidemiological relationship between chronic obstructive pulmonary disease and sleep apnea syndrome in Iran. Prevalence rates for sleep apnea, chronic obstructive pulmonary disease and current asthma were 4.98\%, 5.7\% and 3.1\%, respectively. Logistic regression showed independent associations between sleep apnea and chronic obstructive pulmonary disease. ${ }^{5}$

Present study revealed that maximum of subjects $(68 \%)$ were having average sleep quality of life (SQOL), on the other hand only $32 \%$ were having good SQOL and no patient had poor SQOL.

On contradictory to this finding, De Sajal (2009)conducted a study on Subjective assessment of quality of sleep in chronic obstructive pulmonary disease patients in India. Forty clinically stable COPD patients were recruited from outpatient department. subjective quality of sleep was measured by Pittsburgh Sleep Quality Index (PSQI). All subjects had poor sleep quality with the median global Pittsburgh Sleep Quality Index score. There was no correlation of global PSQI score with severity of COPD observed. ${ }^{6}$ 
The present study revealed that out of 100 stable COPD patients, $60 \%$ patients of high risk obstructive sleep apnea had average sleep quality of life while only $12 \%$ showed good sleep quality of life as compared to $8 \%$ low risk group had average sleep quality of life and $20 \%$ had good sleep quality of life. There was a significant association between obstructive sleep apnea risk and sleep quality of life $(\mathrm{p}=0.00)$.

The findings were supported by a cross-sectional descriptive study conducted by Zohal Ali Mohammad et.al (2013) on 139 COPD patients in a chest clinic of a university hospital in Iran. Analysis of data showed that quality of sleep was significantly correlated with quality of life $(\mathrm{P}<0.001)$. About half of the patients were at high risk for developing obstructive sleep apnea. Findings revealed that patients with overlap syndrome had worse quality of sleep compared to patients without it $(\mathrm{P}<0.001)^{7}$

The present study revealed that variables i.e. gender, martial status, working status, smoking habit and alcohol consumption, duration of treatment, presence of other chronic illness, BMI and neck circumference were found out to be statistically associated with obstructive sleep apnea risk of COPD patients $(\mathrm{p}<0.05)$.

It was supported by a study conducted by Yan li et.al in China. They sequentially recruited 2,749 OSA patients treated in hospital from 2008 to 2013. BMI and WHR increased with OSA severity, in an approximately linear manner, upon univariate plotting. The Pearson correlation coefficients for BMI and WHR were $0.416(\mathrm{P}<0.001)$ and $0.229(\mathrm{P}<0.001)$, respectively. ${ }^{8}$

Community-based studies conducted in the United States and abroad have since confirmed that excess body weight is uniformly associated with a graded increase in obstructive sleep apnea prevalence. Moreover, longitudinal data from the Sleep Heart Health Study, Wisconsin Sleep Cohort Study, and the Cleveland Family Study showed that an increase in body weight over time can certainly accelerate the progression of obstructive sleep apnea. ${ }^{8}$

Epidemiologic studies have confirmed the higher prevalence of obstructive sleep apnea in men. Epidemiologic investigations also showed that current smoking is associated with a higher prevalence of snoring and obstructive sleep apnea. Even exposure to second-hand smoke has been independently linked with habitual snoring. ${ }^{8}$

Ingestion of alcohol before sleep has been shown to increase upper airway collapsibility and precipitate obstructive apneas and hypopneas during sleep. ${ }^{8}$

\section{Conclusion:-}

It was concluded that risk for obstructive sleep apnea was higher in COPD patients and maximum number of the COPD patients had average sleep quality of life. The study shows that there was significant association between obstructive sleep apnea risk and sleep quality of life. $(<0.05)$. Variables like gender, marital status, working status, smoking habits, alcohol consumption, BMI and neck circumference were found to be associated with obstructive sleep apnea risk. $(<0.05)$.

\section{Recommendations:-}

1. The study can be replicated on large sample to validate and generalize its findings.

2. A comparative study can be conducted on obstructive sleep apnea risk and sleep quality of life in COPD patients and healthy individuals.

3. A descriptive study can be conducted to assess the obstructive sleep apnoea risk on different population i.e. stroke patients, elderly patients and obese individuals.

4. A protocol can be developed in hospitals to assess the OSA among every COPD patients and specific interventions can be planned to prevent and treat OSA among COPD patients.

\section{Limitations:-}

1. The sample size of the study was only 100 hence it was difficult to make a broad generalization.

2. Convenience sampling was used to select the target population, which restricted the generalization of the study to the particular setting.

3. The sleep pattern in this study was evaluated using self-reports, and there was no validation by more objective measures such as polysomnography. 
4. This is a descriptive study, and, therefore, it was not possible to evaluate the effects of the variables with time. Thus, longitudinal studies are needed in order to investigate possible risk factors for a poor sleep pattern.

\section{Nursing Implications:-}

The findings of this study can be utilised in all the domains of nursing i.e. nursing practice, nursing education and nursing administration and the implications are:

\section{Nursing Practice:-}

1. Health personnel should have knowledge about obstructive sleep apnoea risk in COPD patients. Hence, they can prevent the complications by assessing the sign and symptoms of OSA and providing education to individuals.

2. The assessment of the risk of OSA and referral for appropriate treatment should be a part of routine nursing clinical workup.

3. Nursing interventions should also be planned to improve sleep quality of COPD patients.

\section{Nursing Education:-}

Ensure that nursing students should know about the prevalence of obstructive sleep apnea in COPD patients, factors associating with OSA and sleep quality of life among COPD patients and it should be incorporated into the nursing curriculum.

\section{Nursing Administration:-}

1. Collaborate with hospital authorities in formulating policies to assess risk for obstructive sleep apnoea in COPD patients and its prevention.

2. Administrative support should be provided to conduct in-service educational program for the nursing personnel regarding obstructive sleep apnea risk, screening of OSA and its prevention in COPD patients.

\section{References:-}

1. Britt H. A decade of Australian general practice activity. General practice series no.34. Sydney: Sydney university Press; 2013; 145-49

2. Sumer S. Choudhary. Sleep effects on breathing and respiratory diseases. Lung India. 2009 Oct-Dec; 26(4): $117-22$.

3. Shah N, Roux F, Mohsenin V. Improving Health-Related Quality of Life in Patients with Obstructive Sleep Apnea: What are the Available Options. Treatment of Respiratory Medicine. 2006; 5(4): 235-244.

4. Feinsilver Steven et.al. Obstructive Airway Disease and Obstructive Sleep Apnea: Effect of Pulmonary Function. Lung. Available at PMC 2012 Aug 13. 2011 Feb; 189(1): 37-41.

5. Mohammad Golshan et.al Correlation between chronic obstructive pulmonary disease and obstructive sleep apnea in a general population in Iran.J Res Med Sci. 2011; 16(7): 885-889.

6. LW hang, JY Hsu, CJ Chang, HC Wang, SL Cheng and CH Lin et al. Predictive factors warrant screening for obstructive sleep apnea in COPD: a Taiwan National survey. International Journal of Chronic Obstructive Pulmonary Disease. 2014; 665-73

7. Sajal De. Subjective assessment of quality of sleep in chronic obstructive pulmonary disease patient and its relationship with associated depression.Lung India, Vol. 29, No. 4, October-December, 2012; 332-35.

8. Dyken ME, Afifi AK, Lin-Dyken DC. Sleep-related problems in neurologic diseases. Chest 141: 2012; 528-44. 